Open Access

\title{
miR-29b-3p regulated osteoblast differentiation via regulating IGF-1 secretion of mechanically stimulated osteocytes
}

\author{
Qiangcheng Zeng ${ }^{1+}$, Yang Wang ${ }^{2,3+}$, Jie Gao ${ }^{1,4}$, Zhixiong Yan², Zhenghua Li ${ }^{1}$, Xianqiong Zou ${ }^{2}$, Yanan Li \\ Jiahui Wang ${ }^{2}$ and Yong Guo ${ }^{1,2^{*}}$
}

\author{
*Correspondence: guoyong74@163. \\ com \\ ${ }^{\dagger}$ Qiangcheng Zeng and Yang Wang \\ contributed equally to this work. \\ 'key laboratory of Functional \\ Bioresource Utilization in University \\ of Shandong, Shandong Key \\ Laboratory of Biophysics, Dezhou \\ University, Dezhou 253023, China \\ ${ }^{2}$ Department of Biomedical \\ Engineering, College of \\ Biotechnology, Guilin Medical \\ University, No. 1 Zhiyuan Road, \\ Lingui District, Guilin City 541100 , \\ Guangxi, China \\ Full list of author information is \\ available at the end of the article
}

\begin{abstract}
Background: Mechanical loading is an essential factor for bone formation. A previous study indicated that mechanical tensile strain of 2500 microstrain $(\mu \varepsilon)$ at $0.5 \mathrm{~Hz}$ for $8 \mathrm{~h}$ promoted osteogenesis and corresponding mechanoresponsive microRNAs (miRs) were identified in osteoblasts. However, in osteocytes, it has not been identified which miRs respond to the mechanical strain, and it is not fully understood how the mechanoresponsive miRs regulate osteoblast differentiation.
\end{abstract}

Methods: Mouse MLO-Y4 osteocytes were applied to the same mechanical tensile strain in vitro. Using molecular and biochemical methods, IGF-1 (insulin-like growth factor-1), PGE2 (prostaglandin E2), OPG (osteoprotegerin) and NOS (nitric oxide synthase) activities of the cells were assayed. MiR microarray and reverse transcriptionquantitative polymerase chain reaction (RT-qPCR) assays were applied to select and validate differentially expressed miRs, and the target genes of these miRs were then predicted. MC3T3-E1 osteoblasts were stimulated by the mechanical tensile strain, and the miR-29b-3p expression was detected with miR microarray and RT-qPCR. Additionally, the effect of miR-29b-3p on IFG-1 secretion of osteocytes and the influence of conditioned medium of osteocytes transfected with miR-29b-3p on osteoblast differentiation were investigated.

Results: The mechanical strain increased secretions of IGF-1 and PGE2, elevated OPG expression and NOS activities, and resulted in altered expression of the ten miRs, and possible target genes for these differentially expressed miRs were revealed through bioinformatics. Among the ten miRs, miR-29b-3p were down-regulated, and miR-29b-3p overexpression decreased the IGF-1 secretion of osteocytes. The mechanical strain did not change expression of osteoblasts' miR-29b-3p. In addition, the conditioned medium of mechanically strained osteocytes promoted osteoblast differentiation, and the conditioned medium of osteocytes transfected with miR-29b-3p mimic inhibited osteoblast differentiation.

Conclusions: In osteocytes (but not osteoblasts), miR-29b-3p was responsive to the mechanical tensile strain and regulated osteoblast differentiation via regulating IGF-1 secretion of mechanically strained osteocytes.

Keywords: Mechanical tensile strain, Osteocyte, Osteoblast differentiation, miRNA microarray 


\section{Introduction}

Mechanical stimulation plays an essential role in the metabolic balance of bone. Physiological loading can induce bone formation, whereas a lack of loading or excessive loading leads to bone resorption [1-4].

As the dominant cells in bone tissue, osteocytes respond to mechanical stimulation, sense and integrate mechanical stimuli into biochemical signals to regulate both bone formation and resorption [5]. Previous studies mainly focused on osteocytes' response to fluid shear stress which inhibits osteocytes apoptosis and promotes survival by modulating the $\mathrm{Bcl}-2 / \mathrm{Bax}$ expression ratio, enhances expression levels of $\mathrm{NO}$ and PGE2, and increases COX2 and the OPG/RANKL ratio, playing a dominant role in regulating the activities of both osteoblasts and osteoclasts [6-8], thus regulating bone reconstruction and remodeling. However, how osteocytes convert the mechanical stimulation into a biological signal and regulate bone formation (activity of osteoblasts) or resorption (activity of osteoclasts) remains not fully elucidated.

MiRs are small non-coding, single-strand RNAs, which control gene expression by targeting to $3^{\prime}$ untranslated regions of mRNA resulting in translational repression or degradation [9]. It was previously found that miR plays a pivotal role in bone formation [10], and many miRs which regulate bone formation have been identified [10, 11]. Some mechanoresponsive miRs were recently identified, they played significant roles in bone formation. For example, miR-33-5p and miR-132 are responsive to mechanical loading and regulate osteogenesis via targeting Hmga2 and mTOR signaling pathway, respectively $[12,13]$. Our previous study confirmed that a mechanical tensile strain of $2500 \mu \varepsilon$ at $0.5 \mathrm{~Hz}$ for $8 \mathrm{~h}$ promoted osteogenesis and mechanoresponsive miRs in osteoblasts were identified [14]. The study urged us to investigate osteocytes' response to the mechanical tensile strain and to search for mechanoresponsive miRs of osteocytes.

miR-29b regulated osteoblast differentiation (in MC3T3 osteoblasts, miR-29b overexpression promotes osteogenic differentiation) [15], and IGF-1 was confirmed to be a target gene of miR-29b [16, 17]. We speculated that miR-29b was responsive to mechanical strain applied to osteocytes and involved in osteoblast differentiation. However, the mechanism by miR-29b osteocytes convert a mechanical signal into a biological signal and regulate osteoblast differentiation has not been fully elucidated.

In this study, the osteocytes' biological response to a mechanical tensile strain of $2500 \mu \varepsilon$ at $0.5 \mathrm{~Hz}$ for $8 \mathrm{~h}$ was investigated, and some novel mechanosensitive miRs were selected. In addition, the involvement of miR-29b in osteocytes' response to mechanical strain and osteoblast differentiation were studied.

\section{Methods}

\section{Cell culture}

A mouse MLO-Y4 osteocyte cell line (provided by JENNIO Biological Technology, Guangzhou, China) was cultured in dishes with $\alpha$-MEM medium ( $\alpha$-MEM, Invitrogen), containing 10\% FBS and 1\% penicillin. Then the cells were transferred to mechanical loading dishes that were reformed from cell culture dishes (Nalge Nunc International).

Mouse MC3T3-E1 osteoblastic cells (JENNIO Biological Technology, Guangzhou) were cultured with the same medium as mentioned above. 


\section{Application of mechanical strain}

At confluence, the medium was renewed with FBS-free medium, then the MLO-Y4 cells were stimulated with mechanical tensile strain of $2500 \mu \varepsilon$ at $0.5 \mathrm{~Hz}$ for $8 \mathrm{~h}$ by a four-point bending device, as previously described [18].

\section{Enzyme-linked immunosorbent assay (ELISA)}

Following mechanical tensile strain, the expression levels of IGF-1 and PGE2 in the collected culture supernatant were detected using an IGF-1 ELISA kit (Boster Bioengineering Co., Ltd., Wuhan China) and PGE2 EIA kit (Cayman Chemical, Michigan USA), according to the manufacturers' instructions. An ELISA reader (Thermo Scientific Multiskan FC ELISA Reader, Rockford, IL, USA) was used to measure the absorbances at $450 \mathrm{~nm}$ and $420 \mathrm{~nm}$ respectively, with the results presented as the content of changes, compared to the unstrained control.

\section{Western blot}

Following mechanical tensile strain, cell lysates were prepared in RIPA lysis buffer (Beijing Solarbio Science \& Technology, Co. Ltd., Beijing, China). Protein in cell lysates was quantified using the BCA method. Equal amounts of proteins were separated by electrophoresis on a polyacrylamide gel containing $0.15 \%$ SDS, then transferred onto PVDF membranes (Millipore, Bedford, MA, USA). After blocking with 5\% skim milk and incubation with primary antibodies overnight at $4{ }^{\circ} \mathrm{C}$, the membranes were incubated with horseradish peroxidase conjugated secondary antibody. The immunoreactive bands were visualized using an ECL detection kit (7 sea biotech Co. Ltd., Shanghai, China). $\beta$-actin in cell lysates was used as a loading control. Data were normalized against those of corresponding optical density of $\beta$-actin.

\section{Detection of NOS activity}

After mechanical tensile strain of MLO-Y4 for $8 \mathrm{~h}$, cells were collected and bathed gently in an ultrasonic processor (UP 400S, Hielscher, Germany) for 2 min. NOS activity was measured using a colorimetric method based on NOS ability to catalyze L-Arg and molecular oxygen to generate NO, and generated NO produces colored compounds with nucleophiles. The optical densities at $530 \mathrm{~nm}$ wavelength were obtained using an ELISA reader (Thermo Scientific Multiskan FC ELISA Reader) and activities of NOS were calculated according to the calibration formula provided in the instructions.

\section{Microarray and RT-qPCR validation of miR}

The RiboArray miDETECT mouse array (Ribobio Co., Guangzhou, China) and RT-qPCR were used to detect and validate the miR expression levels in MLO-Y4 cells. The miR expression levels of the mechanically strained group were compared with the unstrained group.

Briefly, total RNA extraction and miR enrichment procedures were performed using the Trizol method and an mirVana miR Isolation kit (Ambion Life Technologies, Carlsbad, CA, USA), according to the manufacturer's instructions. Target labeling, hybridization, imaging and data processing were performed, according to the manufacturer's instructions using a RiboArray miDETECT mouse array (Ribobio Corporation) which contained all 
mouse miRNAs of Sanger miRBase 19. Data were acquired using Agilent Feature Extraction software version 10.7 (Agilent, Palo Alto, CA, USA) [16, 17]. Further data analyses were performed using GeneSpring GX 10.0 software (Agilent, Palo Alto, CA, USA). Following microarray detection, expression levels of 40 miRs with significant differences were validated by RT-qPCR at Ribobio Co., Ltd. in Guangzhou. The miDETECT A Track Uni-Reverse Primers and miDETECT A Track miRNA Forward Primers (specific primers) for RT-qPCR of these miRs were provided by Ribobio Corporation (Ribobio Co., Ltd. Guangzhou, China). Poly(A) tailing, reverse transcription and qPCR were performed successively using the miDETECT A Track miRNA qRT-PCR Starter Kit (Ribobio Co., Ltd). The reactions were incubated in a 96 -well optical plate at $95^{\circ} \mathrm{C}$ for $20 \mathrm{~s}$, followed by $40 \mathrm{cy}$ cles of $10 \mathrm{~s}$ at $95^{\circ} \mathrm{C}, 20 \mathrm{~s}$ at $60^{\circ} \mathrm{C}$, and $10 \mathrm{~s}$ at $70^{\circ} \mathrm{C}$. Expression analysis was performed in triplicate for each sample. U6 was used as the normalization control. The miR expression levels were quantified using a CFX 96 system (Bio-Rad Laboratories, Hercules, CA).

\section{miRs transfection and preparation of osteocytes' conditioned medium}

MLO-Y4 osteocyte cells, at 70\% confluence, were transfected by miR-29b-3p mimic, miR-29b-3p inhibitor and miR control (Ribobio Co., at a final concentration of $50 \mathrm{nM}$ ) respectively, using the riboFect CP Transfection Kit (Ribobio Co.) according to the manufacturer's method.

The cells were carefully washed in serum-free medium to remove proteins from the bovine serum supplement and then incubated in fresh serum-free medium for $24 \mathrm{~h}$ (stimulated with mechanical tensile strain or not). After centrifugation at $3000 \mathrm{~g}$ for $12 \mathrm{~min}$, the conditioned medium was collected and prepared for the next experiment.

\section{Detection of osteoblastic differentiation}

MC3T3-E1 osteoblastic cells at confluence were carefully washed in serum-free medium, then the cells were cultured in osteocytes' conditioned medium for $24 \mathrm{~h}$. Cells were harvested, washed with a phosphate buffer solution (PBS), and lysed with a lysis buffer (Beijing Solarbio Science \& Technology, Co. Ltd). The ALP activity of the lysates was measured with the ALP Activity Assay Kit (Nanjing Jiancheng Biotechnology Co. Ltd., China) at $25^{\circ} \mathrm{C}$ according to the provider's protocol. The bone morphogenetic protein 2 (BMP-2) of the osteoblastic cells was assayed with an ELISA kit (Elabscience Biotechnology Co., Ltd., Wuhan, China), according to the manufacturer's instructions mentioned above.

cDNA was synthesized using the TIANScript RT kit (Tiangen Biotech Co., Ltd., Beijing, China), then the Runx 2 mRNA was detected using SYBR Green I PCR Mix (Beijing CoWin Biotech Co., Ltd., Beijing, China) according to the manufacturer's method. The PCR amplification reaction included a denaturation step at $94{ }^{\circ} \mathrm{C}$ for $3 \mathrm{~min}$ followed by 40 cycles of $94^{\circ} \mathrm{C}$ for $15 \mathrm{~s}$, annealing at $60^{\circ} \mathrm{C}$ for $30 \mathrm{~s}$, and extension at $72^{\circ} \mathrm{C}$ for $30 \mathrm{~s}$. Relative expression was normalized to mRNA levels of GAPDH using the $2^{-\Delta \Delta} \mathrm{Cq}$ method.

\section{Bioinformatics analysis}

miRWalk2.0 (http://zmf.umm.uni-heidelberg.de/apps/zmf/mirwalk2/), MicroRNA. 
org (www.microrna.org/) and TargetScan (www.targetscan.org/) were applied to predict target genes for these differently expressed miRs. The same target genes for one corresponding miR presented in three online databases were considered as potential targets.

\section{Statistical analysis}

All data are presented as the mean \pm standard deviation from three separate experiments $(n=5$ or 6$)$. Data were tested for normal distribution using the Shapiro-Wilk test and differences between groups were analyzed using one-way analysis of variance and determined by the least significant difference test. Statistical analysis was performed using SPSS software (version 18; SPSS, Inc., Chicago, IL, USA). $P<0.05$ was considered to indicate a statistically significant difference.

\section{Results}

We first investigated osteocytes' response to a physiological mechanical tensile strain of $2500 \mu \varepsilon$ at $0.5 \mathrm{~Hz}$ for $8 \mathrm{~h}$. As shown in Fig. 1, the results of ELISA for IGF-1 and PGE2, western blot for OPG and biochemical method for NOS activity were all changed after applying the mechanical tensile strain to MLO-Y4 cells. All four factors play important roles in bone formation: IGF-1 is widely distributed and plays a significant role in bone development via endocrine, paracrine and autocrine by combining with its ligands [23, 24], PGE2 is a necessary factor in gap junction mediated intercellular communication

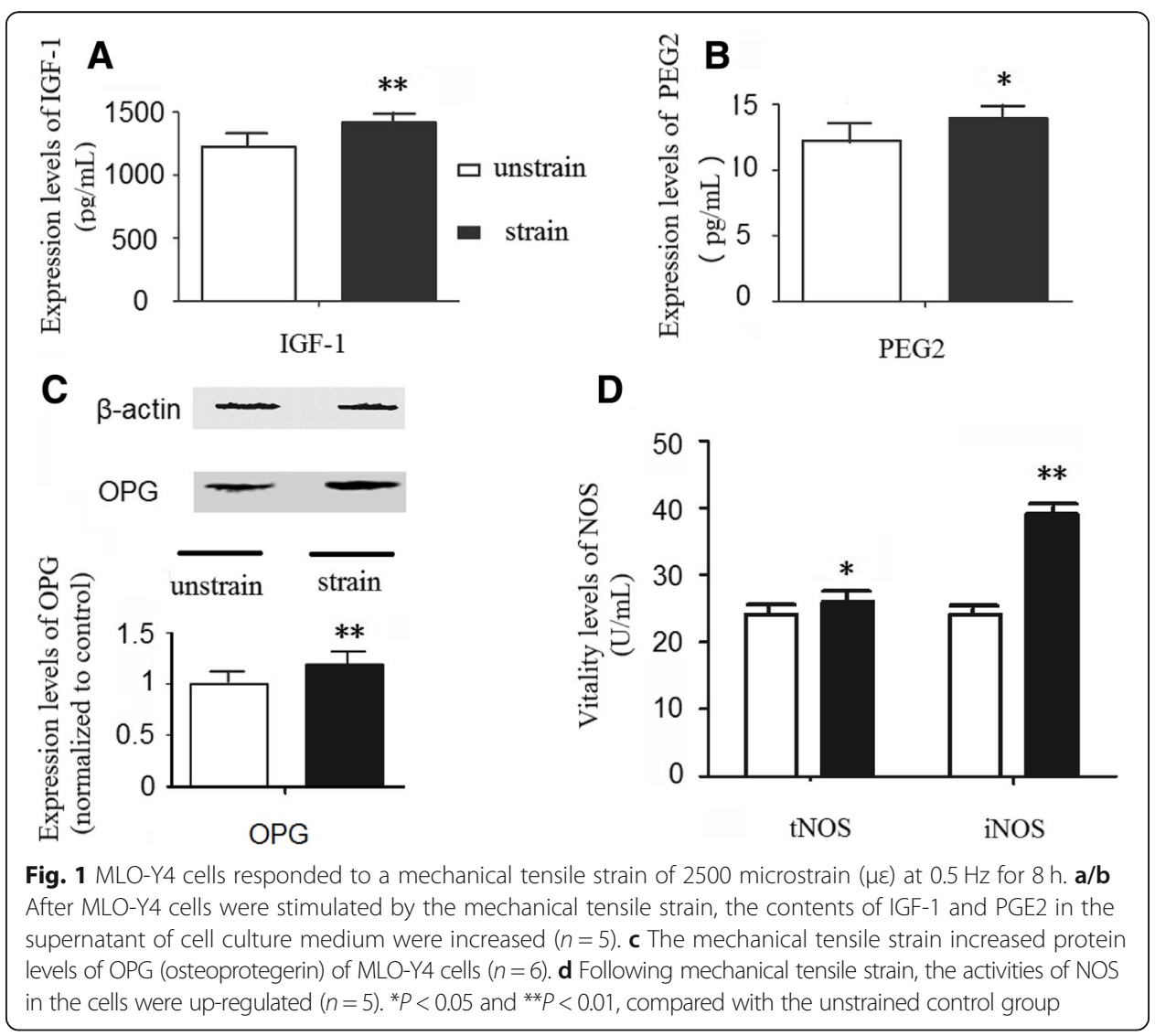


in osteocytes [25]. OPG has been reported to promote bone formation and its absence caused onset of osteoporosis and arterial calcification in mice [26], and NOS is closely associated with the expression level of NO, which could regulate the activity of osteoblasts [27].

Next, we screened and validated 10 miRs' response to the mechanical tensile strain applied to MLO-Y4 cells. A total of 40 miRs were differentially expressed through a miR microarray screen (Fig. 2). Then, using qPCR, the 40 miRs were validated further. The results of miR microarray and qRT-PCR indentified 10 miRs that were mechanoresponsive miRs: miR-713, miR-706, miR-703, miR-574-3p, miR-467b-3p, miR-466i-5p, miR-466f-5p and miR -208a-3p were up-regulated and miR-29b-3p and miR-361-3p were down-regulated, compared to the unstrained group (Fig. 2, Fig. 3a).

Bioinformatics was applied to predict target genes for these differently expressed miRs. The results indicated that several target genes were in correlation with bone formation (Table 1). In all of the putative target genes, we found 29 genes that correlated with bone formation and might play important roles in bone formation. Of all the target genes, IGF-1 attracted our attention the most: IGF-1 was a target gene of miR-29b-3p, confirmed by previous studies $[16,17]$. In our study, the expression level of IGF-1 was increased (Fig. 1) while the expression level of miR-29b-3p was decreased in mechanically strained osteocytes (Fig. 3a), indicating that miR-29b-3p was likely to regulate bone formation via targeting IGF-1.

Finally, we went on study to investigate the involvement of miR-29b-3p in osteocytes' response to mechanical strain and the effect of the miR on osteoblast differentiation. We found that mechanical tensile strain down-regulated miR-29b-3p expression of MLO-Y4 cells, not MC3T3-E1 cells, and miR-29b-3p inhibited IGF-1 secretion of osteocytes (Fig. 3c). The miR-29b-3p mimic transfection resulted in over-expression of the miR in MLO-Y4 cells, which caused a reduction of IGF-1 levels in osteocytes' culture supernatant, and miR-29b-3p inhibitor transfection, which resulted in low miR-29b-3p expression, and increased the IGF-1 secretion (Fig. 3b, Fig. 4a). In addition, the conditioned medium of MLO-Y4 cells transfected with miR-29b-3p mimic decreased ALP activity, and reduced expression of BMP-2 and Runx 2 in MC3T3-E1 cells (Fig. 4 b-d). In contrast, the conditioned medium of MLO-Y4 cells transfected with miR-29b-3p

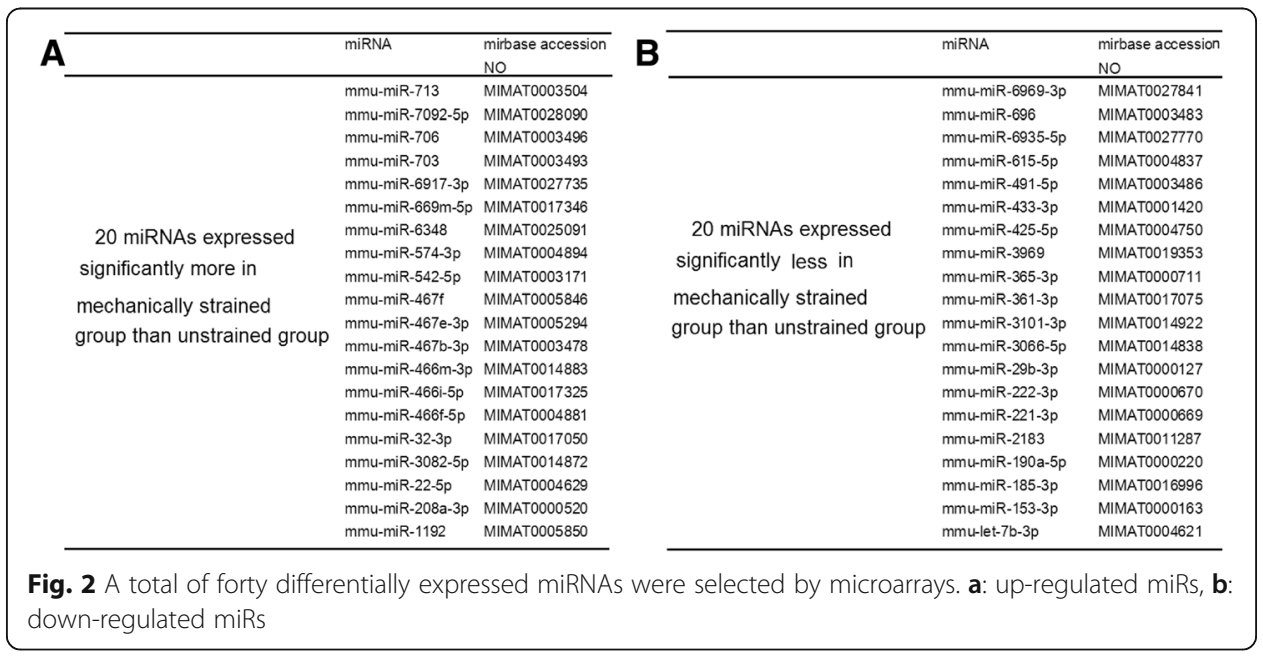




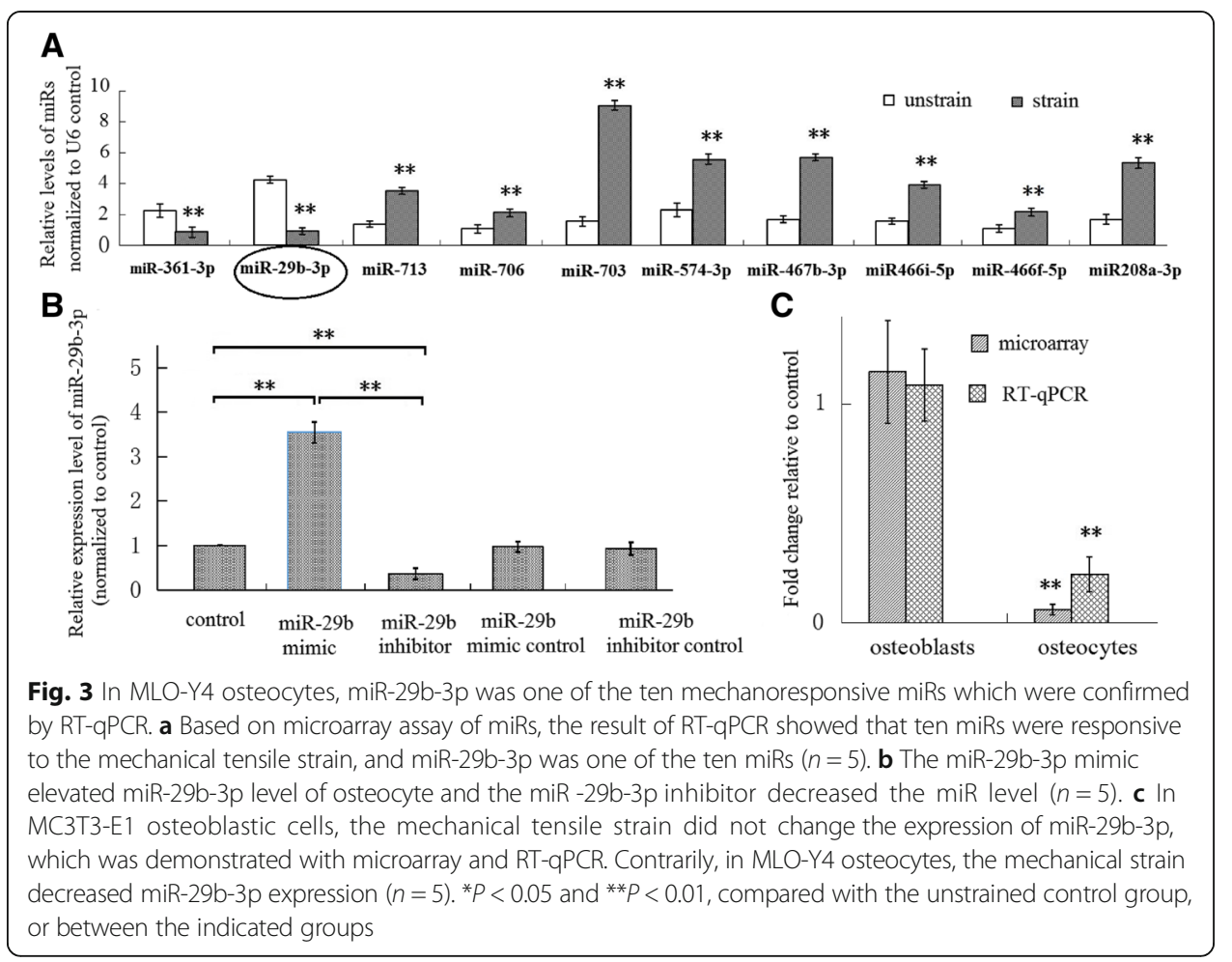

inhibitor increased ALP activity and enhanced expression of BMP-2 and Runx 2 in MC3T3-E1 cells (Fig. 4 b-d). These results indicated that miR-29b-3p regulated osteoblast differentiation via osteocyte secretion.

\section{Discussion}

In this study, the results indicated that osteocytes responded to a mechanical tensile strain of $2500 \mu \varepsilon$ at $0.5 \mathrm{~Hz}$ for $8 \mathrm{~h}$. Following osteocytes' response to this mechanical loading, 10 mechanoresponsive miRs in MLO-Y4 osteocytes were identified, bioinformatics analysis revealed the possible target genes of these miRs, and 29 target genes were correlated with bone formation.

Among the mechanoresponsive miRs and their target genes, the relationship between miR-29b-3p (1 of 10 mechanoresponsive miRNAs) and its target gene, IGF-1, promoted us to find the regulatory relation of osteocytes to osteoblasts under the same mechanical stimulation. In this study, in MC3T3-E1 osteoblastic cells, miR-29b-3p was not involved in osteoblasts' response to the mechanical strain, and the conditioned medium of the mechanically strained osteocytes promoted osteoblast differentiation of MC3T3-E1 cells. Additionally, the over-expression of miR-29b-3p inhibited osteoblast differentiation via reducing the IGF-1 level in the conditioned culture medium of MLO-Y4 osteocytes which was used to culture MC3T3-E1 cells. During the response to mechanical strain, miR-29b-3p had no direct effect on osteoblasts, and the miR regulated osteoblast differentiation via a mediator: osteocytes, because the mechanical strain up-regulated the cells' miR-29b-3p, which reduced IGF-1 secretion of MLO-Y4 osteocytes. 
Table 1 Predicted target genes of the miRs

\begin{tabular}{|c|c|c|}
\hline miRNA & Annotation and Reference & RefseqID \\
\hline \multirow[t]{6}{*}{ mmu-miR-29b-3p } & IGF-1, Insulin-like Growth Factor-1[23, 24] & NM_000076.6 \\
\hline & Grip 1, Glutamate Receptor-Interacting Protein 1 [28] & NM_028736 \\
\hline & Dtx4, deltex 4, E3 ubiquitin ligase [29] & NM_172442 \\
\hline & Smad5, SMAD family member 5 [30] & NM_001164041 \\
\hline & Wnt2b, wingless-type MMTV integration site family, member 2B [31] & NM_009520 \\
\hline & Rptor, regulatory associated protein of MTOR complex 1 [32] & NM_028898 \\
\hline mmu-miR-361-3p & Map3k9, mitogen-activated protein kinase kinase kinase 9 [33] & NM_001174107 \\
\hline \multirow[t]{2}{*}{ mmu-miR-713 } & Rag1, recombination activating gene 1 [34] & NM_009019 \\
\hline & Gprc5a, G protein-coupled receptor, family C, group 5, member A [35] & NM_181444 \\
\hline \multirow[t]{3}{*}{ mmu-miR-706 } & Dusp3, dual specificity phosphatase 3 [36] & NM_028207 \\
\hline & Ncoa1, nuclear receptor coactivator $1[37]$ & NM_010881 \\
\hline & Nkiras1, NFKB inhibitor interacting Ras-like protein 1[38] & NM_023526 \\
\hline \multirow[t]{2}{*}{ mmu-miR-703 } & ZnT4, solute carrier family 30 [39] & NM_011774 \\
\hline & Smad5, SMAD family member 5 [30] & NM_001164041 \\
\hline \multirow[t]{3}{*}{ mmu-miR-574-3p } & Tgfbr3, transforming growth factor, beta receptor III [40] & NM_011578 \\
\hline & Dicer1, dicer 1, ribonuclease type III [41] & NM_148948 \\
\hline & Pdk1, pyruvate dehydrogenase kinase, isoenzyme 1 [42] & NM_172665 \\
\hline \multirow[t]{3}{*}{ mmu-miR-467b-3p } & Runx2, runt-related protein 2 [43] & NM_001145920 \\
\hline & Pdgfra, platelet derived growth factor receptor alpha [44] & NM_001083316 \\
\hline & Mapk10, mitogen-activated protein kinase 10 [45] & NM_009158 \\
\hline \multirow[t]{3}{*}{ mmu-miR-466i-5p } & Atm, ataxia telangiectasia mutated [46] & NM_007499 \\
\hline & Rgs2, regulator of G-protein signaling 2 [47] & NM_009061 \\
\hline & Smo, smoothened, frizzled class receptor [48] & NM_176996 \\
\hline \multirow[t]{3}{*}{ mmu-miR-466f-5p } & Rcan1, regulator of calcineurin 1[49] & NM_001081549 \\
\hline & Clock, circadian locomotor output cycles kaput [50] & NM_007715 \\
\hline & Aff3, AF4/FMR2 family, member 3 [51] & NM_010678 \\
\hline \multirow[t]{4}{*}{ mmu-miR-208a-3p } & ॥6ra, interleukin 6 receptor [52] & NM_010559 \\
\hline & Acvr1C, activin A receptor type 1 C [40] & NM_001111030 \\
\hline & Sox6, SRY-Box 6 [40] & NM_011445 \\
\hline & Sox5, SRY-Box 5 [40] & NM_011444 \\
\hline
\end{tabular}

For the target genes which are involved in bone formation, the references are provided

Osteocytes in the lacunar-canalicular system of the bone are mainly mechanosensory cells in bone tissue. They transduce mechanical stimulation into biomechanical signals and regulate bone remodeling by regulating the activities of osteoblasts and osteoclasts $[19,20]$. In this study, during the response to mechanical strain, miR-29b-3p had no direct effect on osteoblasts, and the miR regulated osteoblast differentiation via a mediator: osteocytes, because mechanical strain up-regulated the cells' miR-29b-3p, which reduced IGF-1 secretion of MLO-Y4 osteocytes.

It has been reported that osteocytes are responsive to fluid shear stress, microgravity and fluid flow [21, 22]. The results of our study suggested that osteocytes could also respond to mechanical tensile strain and regulate osteoblast differentiation via miR-29b-3p regulating of IGF-1 secretion, which will shed some light on how osteocytes regulate osteogenesis. 

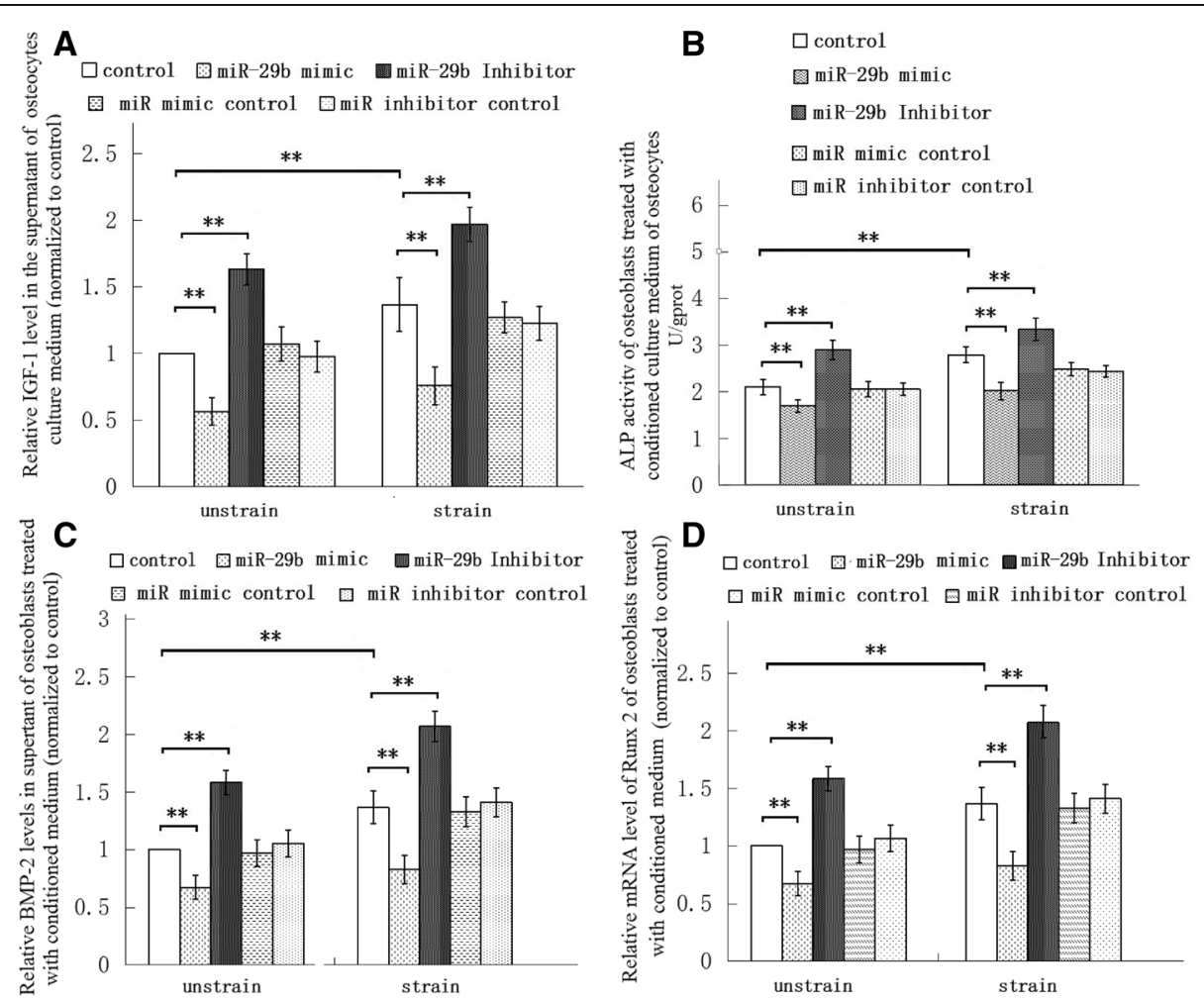

Fig. 4 miR-29b-3p regulated IGF-1 secretion of MLO-Y4 osteocytes, and after the osteocytes were transfected with miR-29b-3p mimic or miR-29b-3p inhibitor, the osteocytes' conditioned culture medium influenced osteoblastic differentiation of MC3T3-E1 cells. a miR-29b-3p mimic decreased IGF-1 secretion of osteocytes, and the miR inhibitor increased the IGF-1 secretion $(n=6)$. The conditioned medium of osteocytes treated with miR-29b-3p mimic reduced ALP activity of osteoblasts (b), BMP-2 protein (c) and Runx 2 mRNA (d) levels of osteoblasts $(n=6)$. In contrast, the conditioned medium of osteocytes treated with miR-29b-3p inhibitor enhanced ALP activity of osteoblasts (b), BMP-2 protein (c) and Runx 2 mRNA (d) levels of osteoblasts $(n=6) .{ }^{*} P<0.05$ and ${ }^{* *} P<0.01$, compared with the unstrained control group, or between the indicated groups

\section{Conclusions}

Osteocytes responded to a cyclic mechanical tensile strain of $2500 \mu \varepsilon$ at $0.5 \mathrm{~Hz}$ for $8 \mathrm{~h}$, mechanoresponsive miRs were discovered in osteocytes, miR-29b-3p was responsive to the mechanical tensile strain and the miR regulated osteoblast differentiation via regulating IGF-1 secretion of osteocytes.

\section{Abbreviations}

BCl-2/Bax: B-cell lymphoma 2/Bax; BMP-2: Bone morphogenetic protein 2; COX2: Cyclooxygenase 2;

ECL: Electrochemiluminescence; ELISA: Enzyme-linked immunosorbent assay; IGF-1: Insulin-like growth factor-1; miR: microRNA; NO: Nitric oxide; NOS: Nitric oxide synthase; OPG: Osteoprotegerin; PGE2: Prostaglandin E2; PVDF: Polyvinylidene fluoride membrane; RANKL: Receptor activator of NF-kB ligand; SDS: Sodium dodecyl sulfate; $\mu \varepsilon$ : Microstrain

\section{Acknowledgements}

The authors are grateful to Xi Lu and Junchen Li at the College of Biotechnology, Guilin Medical University, for their tremendous support.

\section{Funding}

This study was supported by grants from the National Nature Science Foundation of China (No. 31660261, No. 11432016) and the Natural Science Foundation of Guangxi (2018JIA140050, 2016GXNSFAA380322). 
Authors' contributions

QZ designed the study, analyzed data, and prepared conditioned medium; YW generated mechanical tensile strain, performed ELISA and Western blot; ZY performed all RT-qPCR analyses and assisted in the bioinformatics analysis; ZL and JG participated in Western blot and ELISA and performed bioinformatics analysis; YL cultured the cells; JW participated in cell culture and detected NOS activities; YG supervised the experiments and drafted the manuscript. All authors read and approved the final manuscript.

\section{Ethics approval and consent to participate}

Not applicable.

\section{Consent for publication}

Not applicable.

\section{Competing interests}

The authors declare that they have no competing interests.

\section{Publisher's Note}

Springer Nature remains neutral with regard to jurisdictional claims in published maps and institutional affiliations.

\section{Author details}

${ }^{1}$ key laboratory of Functional Bioresource Utilization in University of Shandong, Shandong Key Laboratory of Biophysics, Dezhou University, Dezhou 253023, China. ²Department of Biomedical Engineering, College of Biotechnology, Guilin Medical University, No. 1 Zhiyuan Road, Lingui District, Guilin City 541100, Guangxi, China. ${ }^{3}$ Key Laboratory for Biorheological Science and Technology of Ministry of Education, State and Local Joint Engineering Laboratory for Vascular Implants, Bioengineering College of Chongqing University, Chongqing 400044, China. ${ }^{4}$ Medical Department, Secondary Renmin Hospital of Dezhou, Dezhou 253023, Shangdong, China.

Received: 29 September 2018 Accepted: 23 January 2019

Published online: 05 June 2019

\section{References}

1. Carter DR, Van Der Meulen MC, et al. Mechanical factors in bone growth and development. Bone. 1996;18(1 Suppl):5S-10S

2. Bonnet N, Ferrari SL. Exercise and the skeleton: How it works and what it really does. IBMS BoneKEy. 2010;7:235-48.

3. Boerckel JD, Dupont KM, Kolambkar YM, et al. In Vivo Model for Evaluating the Effects of Mechanical Stimulation on Tissue-Engineered Bone Repair. J Biomech Eng. 2009;131:084502

4. Nagaraja MP, Jo H. The Role of Mechanical Stimulation in Recovery of Bone Loss-High versus Low Magnitude and Frequency of Force. Life. 2014;4:117-30.

5. Schaffler MB, Cheung W, Majeska RJ, et al. Osteocytes: Master Orchestrators of Bone. Calcif Tissue Int. 2013:94:5-24

6. Bakker A, Klein-Nulend J, Burger E. Shear stress inhibits while disuse promotes osteocyte apoptosis. Biochem Biophys Res Commun. 2004;320:1163-8.

7. Bakker AD, Soejima K, Klein-Nulend J, et al. The production of nitric oxide and prostaglandin E2 by primary bone cells is shear stress dependent. J Biomech. 2001;34:671-7.

8. Bonewald LF, Johnson ML. Osteocytes, mechanosensing and Wnt signaling. Bone. 2008;42:606-15.

9. Bartel DP. MicroRNAs: Genomics, Biogenesis, Mechanism, and Function. Cell. 2004;116:281-97.

10. Kapinas K, Delany AM. MicroRNA biogenesis and regulation of bone remodeling. Arthritis Res Ther. 2011;13:220,

11. Qi Z, Liu W, Lu J. The mechanisms underlying the beneficial effects of exercise on bone remodeling: Roles of bonederived cytokines and microRNAs. Prog Biophys Mol Biol. 2016;122:131-9.

12. Wang $H$, Sun $Z$, Wang $Y$, et al. miR-33-5p, a novel mechano-sensitive microRNA promotes osteoblast differentiation by targeting Hmga2. Sci Rep. 2016:6:23170.

13. Qi L, Zhang Y. The microRNA 132 Regulates Fluid Shear Stress-Induced Differentiation in Periodontal Ligament Cells through mTOR Signaling Pathway. Cell Physiol Biochem. 2014;33:433-45.

14. Guo Y, Wang Y, Liu Y, et al. MicroRNA-218, microRNA-191*, microRNA-3070a and microRNA-33 are responsive to mechanical strain exerted on osteoblastic cells. Mol Med Report. 2015;12:3033-8.

15. Li Z, Hassan MQ, Jafferji M, et al. Biological functions of miR-29b contribute to positive regulation of osteoblast differentiation. J Biol Chem. 2009;284:15676-84

16. Yuan L, Zhou C, Lu Y, et al. IFN-Y-mediated IRF1/miR-29b feedback loop suppresses colorectal cancer cell growth and metastasis by repressing IGF1. Cancer Lett. 2015;359(1):136-47.

17. Abonnenc M, Nabeebaccus AA, Mayr U, et al. Extracellular matrix secretion by cardiac fibroblasts: role of microRNA-29b and microRNA-30c. Circ Res. 2013;113(10):1138-47.

18. Liu L, Guo Y, Wan Z, et al. Effects of Phytoestrogen a-ZAL and Mechanical Stimulation on Proliferation, Osteoblastic Differentiation, and OPG/RANKL Expression in MC3T3-E1 Pre-Osteoblasts. Cell Mol Bioeng. 2012;5:427-39.

19. Thi MM, Suadicani SO, Schaffler MB, et al. Mechanosensory responses of osteocytes to physiological forces occur along processes and not cell body and require aVB3 integrin. Proc Natl Acad Sci USA. 2013;110:21012-7.

20. Pajevic PD. Regulation of bone resorption and mineral homeostasis by osteocytes. Ibms Bonekey. 2009;6:63-70

21. Yang X, Sun LW, Wu XT, et al. Impact of shear stress and simulated microgravity on osteocytes using a new rotation cell culture device. Acta Astronaut. 2015;116:286-98.

22. Cherian PP, Sillerjackson AJ, Gu S, et al. Mechanical Strain Opens Connexin 43 Hemichannels in Osteocytes: A Novel Mechanism for the Release of Prostaglandin. Mol Biol Cell. 2005;16:3100-6.

23. Juffer $P$, Jaspers RT, Lips $P$, et al. Expression of muscle anabolic and metabolic factors in mechanically loaded MLO-Y4 osteocytes. Am J Physiol Endocrinol Metab. 2012;302:E389-95. 
24. Sheng $M H$, Zhou $X D$, Bonewald $L F$, et al. Disruption of the insulin-like growth factor-1 gene in osteocytes impairs developmental bone growth in mice. Bone. 2013;52(1):133-44.

25. Cheng B, Kato $Y$, Zhao $S$, et al. PGE2 is essential for gap junction-mediated intercellular communication between osteocyte-like MLO-Y4 cells in response to mechanical strain. Endocrinology. 2001;142:3464-73.

26. Zheng C, Chu P, Wu CC, et al. Association between Increased Serum Osteoprotegerin Levels and Improvement in Bone Mineral Density after Parathyroidectomy in Hemodialysis Patients. Tohoku J Exp Med. 2012;226:19-27.

27. van't Hof RJ, Ralston SH. Nitric oxide and bone. Immunology. 2001;103(3):255-61.

28. Skerry TM. Identification of novel signaling pathways during functional adaptation of the skeleton to mechanical loading: the role of glutamate as a paracrine signaling agent in the skeleton. J Bone Miner Metab. 1999;17:66-70.

29. Harper JA, Yuan JS, Tan JB, et al. Notch signaling in development and disease. Clin Genet. 2003;64:461-72.

30. Proff $P$, Römer P. The molecular mechanism behind bone remodelling: a review. Clin Oral Investig. 2009;13:355-62

31. Qiu W, Andersen TE, Bollerslev J, et al. Patients with high bone mass phenotype exhibit enhanced osteoblast differentiation and inhibition of adipogenesis of human mesenchymal stem cells. J Bone Miner Res. 2007;22:1720-31.

32. Gao J, Cheng TS, Qin A, et al. Glucocorticoid impairs cell-cell communication by autophagy-mediated degradation of connexin 43 in osteocytes. Oncotarget. 2016:7:26966-78.

33. Yan B, Li J, Zhang L. Identification of B cells participated in the mechanism of postmenopausal women osteoporosis using microarray analysis. Int. J. Clin. Exp. Med. 2015;8:1027-34.

34. Schindeler A. Pre-clinical Fracture Repair Studies: Meeting Report from the 31st Annual Meeting of the American Society for Bone and Mineral Research. Ibms Bonekey. 2009;6:481-4

35. Siddappa R, Martens A, Doorn J, et al. cAMP/PKA pathway activation in human mesenchymal stem cells in vitro results in robust bone formation in vivo. Proc Natl Acad Sci. 2008;105:7281-6.

36. Sevetson B, Taylor S, Pan Y. Cbfa1/RUNX2 Directs Specific Expression of the Sclerosteosis Gene (SOST). J Biol Chem. 2004;279:13849-58

37. Takaoka K, Koezuka M, Nakahara H. Telopeptide-depleted bovine skin collagen as a carrier for bone morphogenetic protein. J Orthop Res. 1991;9:902-7.

38. Urist MR. Bone: formation by autoinduction. Science. 1965:150:893-9.

39. Fukada T, Hojyo S, Furuichi T. Zinc signal: a new player in osteobiology. J Bone Miner Metab. 2013;31:129-35.

40. Hoffmann A, Gross G. BMP signaling pathways in cartilage and bone formation. Crit Rev Eukaryot Gene Expr. 2001;11:23.

41. Kobayashi T, Lu J, Cobb BS, et al. Dicer-dependent pathways regulate chondrocyte proliferation,differentiation. Proc Natl Acad Sci USA. 2008; 105:1949-1954

42. Whitfield JF. The solitary (primary) cilium-a mechanosensory toggle switch in bone and cartilage cells. Cell Signal. 2008; 20:1019-24.

43. Lian J, Stein GS. Runx2/Cbfa1: a multifunctional regulator of bone formation. Curr Pharm Des. 2003;9:2677-85.

44. Schatteman GC, Morrison-Graham K, van Koppen A, et al. Regulation and role of PDGF receptor alpha-subunit expression during embryogenesis. Development. 1992:115:123-31.

45. Kilian KA, Bugarija B, Lahn BT, et al. Geometric cues for directing the differentiation of mesenchymal stem cells. Proc Natl Acad Sci. 2010:107:4872-7.

46. Hishiya A, Ito M, Aburatani $\mathrm{H}$, et al. Ataxia telangiectasia mutated (Atm) knockout mice as a model of osteopenia due to impaired bone formation. Bone. 2005:37:497-503.

47. Krishnan V, Moore TL, Ma Y, et al. Parathyroid hormone bone anabolic action requires Cbfa1/Runx2-dependent signaling. Mol Endocrinol. 2003:17:423-35.

48. Day TF, Yang Y. Wnt and hedgehog signaling pathways in bone development. J Bone Joint Surg. 2008;90(Supplement 1):19-24.

49. Srinivasan S, Ausk BJ, Bain SD, et al. Rest intervals reduce the number of loading bouts required to enhance bone formation. Med Sci Sports Exerc. 2015:47:1095-103.

50. Lieben L. Bone: The circadian clock controls bone remodelling. Nat Rev Rheumatol. 2016;12:132-3.

51. van der Meulen MC, Boskey AL. Atypical subtrochanteric femoral shaft fractures: role for mechanics and bone quality. Arthritis Res Ther. 2012:14:220

52. Kwan Tat S, Padrines M, Théoleyre S, et al. IL-6, RANKL, TNF-alpha/LL-1: interrelations in bone resorption pathophysiology. Cytokine Growth Factor Rev. 2004:15:49-60.

\section{Ready to submit your research? Choose BMC and benefit from:}

- fast, convenient online submission

- thorough peer review by experienced researchers in your field

- rapid publication on acceptance

- support for research data, including large and complex data types

- gold Open Access which fosters wider collaboration and increased citations

- maximum visibility for your research: over $100 \mathrm{M}$ website views per year

At $B M C$, research is always in progress. 